\title{
EVALUACIÓN DEL BANCO DE SEMILLAS Y SU IMPORTANCIA EN LA REHABILITACIÓN DE LA VEGETACIÓN DE HUMEDALES DEL CENTRO MÉXICO
}

\author{
Carmen Zepeda-Gómez ${ }^{1,4}$, Antonio Lot ${ }^{2}$, Xanat Antonio-Nemiga ${ }^{3}$ y Javier Manjarrez ${ }^{1}$ \\ 'Universidad Autónoma del Estado de México, Facultad de Ciencias, Toluca, Estado de México, México \\ ${ }^{2}$ Universidad Nacional Autónoma de México, Instituto de Biología, México, D.F., México \\ ${ }^{3}$ Universidad Autónoma del Estado de México, Facultad de Geografía, Toluca, Estado de México, México \\ ${ }^{4}$ Autor para la correspondencia: zepedac@uaemex.mx
}

\begin{abstract}
Resumen: Los métodos de evaluación del banco de semillas tienen una eficiencia particular y cada uno aporta una imagen diferente de la vegetación de un área, no obstante, esta información puede ser muy útil en los programas de restauración de comunidades vegetales anegadas. El objetivo fue comparar cuantitativamente el banco de semilla de la Ciénega de Chignahuapan utilizando dos métodos e inferir si las comunidades vegetales podrían rehabilitarse a partir del mismo. En las zonas estacionalmente inundadas de la ciénega se recolectaron muestras de los $10 \mathrm{~cm}$ más superficiales de sedimento para evaluar el banco de semillas por el método de separación y el de germinación. Los métodos probados difirieron en la composición de especies y en la abundancia de las mismas. El método de separación de semillas predijo una riqueza, diversidad y densidad, significativamente mayor que el método de germinación. Este último mostró una similitud más alta con la vegetación establecida y predijo las especies que germinarán con mayor facilidad del banco. La baja similitud y la inexistencia de relación entre las especies de ambos métodos muestran que cada técnica tiene un poder predictivo diferente, por lo que su aplicación aislada debe evitarse. El banco de semillas de Chignahuapan tiene 60 especies y se caracteriza por la presencia de plantas con alta tolerancia ecológica, baja similitud con la vegetación establecida y la ausencia de acuáticas estrictas anteriormente registradas como elementos típicos de la región. Este banco de semillas puede usarse en futuros programas de restauración de la ciénega con la limitante de restituir principalmente, especies vegetales de estados sucesionales tempranos.
\end{abstract}

Palabras clave: Chignahuapan, hidrófitas, humedales, método de germinación, restauración.

\begin{abstract}
Methods of seed bank evaluation have a particular efficiency and each one provides a different picture of the vegetation, however, this information can be very useful in restoration programs for wetland vegetation. The aim was to compare quantitatively the Chignahuapan seed bank using two methods and infer whether plant communities could be rehabilitated from it. In the seasonally flooded areas of the marsh we collected samples of the top $10 \mathrm{~cm}$ of sediment to evaluate the seed bank by the methods of separation and of germination. The tested methods differ in species composition and in its abundance. Separation method predicted higher richness, diversity and density. Germination method showed a higher similarity to the established vegetation and predicted which species emerge more easily from the bank. The low similarity and the lack of relationship between species of both methods show that each technique has a different predictive power, thus its isolated application should be avoided. Chignahuapan seed bank includes 60 species and is characterized by the presence of plants with high ecological tolerance, low similarity with established vegetation and the absence of strict aquatic plants previously registered as typical elements in the region. This seed bank can be used in future restoration programs of the marsh, with the limitation to restore plant species of early succession stages.
\end{abstract}

Key words: Chignahuapan, germination method, hydrophytes, restoration, wetland.

U na de las estrategias de historias de vida de las plantas que crecen en ambientes estacionalmente inundados es la producción de semillas capaces de sobrevivir enterradas en los sedimentos, y mantenerse en ellos por algún tiempo hasta que las condiciones ambientales permitan su germinación (Leck, 2003). La acumulación progresiva de estas semillas forma depósitos o bancos de semillas (BS) que conservan los genotipos de la vegetación establecida, así 
como los pasados y aquellos dispersados desde otros lugares. Al contener semillas de múltiples generaciones, formadas bajo condiciones ambientales diferentes, los bancos son una fuente potencial de diversidad que cuando se manifiesta determina la estructura de la comunidad vegetal, así como su dinámica y persistencia (Hopfensperger, 2007; Fisher et al., 2009). El conocimiento de la riqueza y abundancia de las semillas del banco, permite predecir que especies de plantas nativas colonizarán el lugar si el ambiente se perturba o si las condiciones hidrológicas se modifican (Cronk y Fennessy, 2001; Cronk y Siobhan, 2001), advierte sobre la posibilidad de futuras infestaciones de especies exóticas e invasoras (Espeland et al., 2010) e informa de las hidrófitas que no están presentes en la vegetación o el banco y que debieran estarlo. De esta forma, el análisis de los BS revela el potencial que se encuentra almacenado en los sedimentos y por ello, ha sido una herramienta útil en los programas de restauración de comunidades vegetales anegadas (Liu et al., 2005; Espeland et al., 2010).

La complejidad estructural de los BS y la necesidad de estimar cuantitativamente la reserva almacenada en el suelo, ha dado lugar a la creación y combinación de varios métodos de análisis, los cuales se concentran en dos grandes grupos: (1) métodos de separación y (2) métodos de germinación (Piudo y Cavero, 2005). En la literatura, los métodos de separación de semillas (MSS) con sus variantes especificas son frecuentes (Baskin y Baskin, 2001; Tsuyuzaki y Masaki, 2001; Ishikawa-Goto y Tsuyuzaki, 2004; McFarland y Shafer, 2011) y consisten en separar las semillas por flotación usando una solución salina, o lavando las muestras de suelo y pasándolas por una serie de tamices de diferente tamaño de malla, a fin de reducir el volumen de la muestra y eliminar la mayor cantidad de materia orgánica, para después separar y observar todas las semillas bajo el microscopio. Son buenos métodos para encontrar semillas grandes y aquellas que están en estado latente, pero requieren de una prueba adicional para evaluar la viabilidad de las diásporas que se extraen (Piudo y Cavero, 2005; McFarland y Shafer, 2011).

Los métodos de germinación (MG) se basan en la emergencia de plántulas desde las muestras de suelo, las cuales se extienden sobre una capa de sustrato y se colocan en condiciones controladas para favorecer la germinación de la mayor cantidad de semillas viables (Bernhardt et al., 2008). Con estos métodos se detecta el componente ecológicamente activo del sedimento (Haag, 1983), incluyendo las semillas pequeñas. Son los más usados para analizar bancos de sedimentos (Van der Valk y Davis, 1978; Haag, 1983; Kimber et al., 1995; de Winton et al., 2000) y algunos autores afirman que son los mejores para estudios de comunidades a gran escala porque, aunque requieren de mayor espacio para su procesamiento, permiten manipular grandes volúmenes de sedimentos (Simpson et al., 1989; Forcella, 1992). Sin embargo, la evaluación del BS a través de estos métodos se ve limitada porque algunos tipos de latencia no pueden suprimirse en el tiempo y espacio experimental al que son sometidas las muestras (Guo et al., 1998).

Se han propuesto procedimientos que fusionan a dos o más técnicas de evaluación del BS (Bernhardt et al., 2008) y en otros casos se han comparado la eficacia entre algunos de ellos (Gross, 1990; Brown, 1992; Ishikawa-Goto y Tsuyuzaki, 2004; Bernhardt et al., 2008; McFarlan y Shafer, 2011). La comparación de los métodos señala que cada técnica tiene una eficiencia particular en la estimación del número de especies presentes y la abundancia relativa de las mismas (Espeland, 2010), de tal forma que la información de la comunidad de plantas que cada uno aporta puede ser diferente. La aplicación de un método en particular estará en función de los objetivos planteados. En trabajos de biodiversidad la evaluación precisa de la composición de especies es más útil que la estimación de la densidad total del banco (Brown, 1992), mientras que para otros, encaminados a la conservación de especies particulares puede ser al contario (Espeland, 2010).

En el presente estudio, el banco de semillas de la Ciénega de Chignahuapan se comparó cuantitativamente utilizando los métodos estándar de separación de semillas y de germinación. La Ciénega de Chignahuapan es un área natural protegida (SEMARNAT, 2002; Pérez y Valdez, 2006) y junto con otras dos zonas inundadas de la región, son los remanentes de los extensos humedales que conformaban a las ciénegas de Lerma. Estas zonas lacustres son altamente diversas; sin embargo, su extensión se ha reducido drásticamente en las últimas décadas por la actividad humana y la sobreexplotación de los mantos freáticos (Antón y Díaz, 2002; Zepeda-Gómez et al., 2012b). El interés por preservar estos ecosistemas y revertir la disminución de la vegetación ocasionada por diferentes factores de perturbación, ha llevado a considerar que los BS que aún se mantienen en las zonas estacionalmente inundadas pueden ser una fuente de recuperación de las comunidades vegetales de la ciénega. En este sentido, el estudio se enfoca en analizar el BS de Chignahuapan comparando la eficiencia que el MSS y el MG tienen para determinar la riqueza y abundancia de las especies que componen el banco de este humedal, y con esta información inferir si a través de estas reservas las comunidades de hidrófitas podrían rehabilitarse.

\section{Materiales y métodos}

Zona de estudio. La Ciénegas de Chignahuapan se ubica en el curso alto de la Cuenca alta del río Lerma, a 26 kilómetros de la capital del Estado de México, sus coordenadas extremas son $19^{\circ} 08^{\prime}-19^{\circ} 09^{\prime}$ latitud norte y $99^{\circ} 29^{\prime}$ - $99^{\circ}$ 31' longitud oeste (Figura 1), cubre una extensión aproximada de 596 ha y se encuentra rodeada de áreas de actividad agrícola intensiva. Se trata de un humedal herbáceo cuyas poblaciones vegetales se distribuyen en función del nivel de inundación. Está formado por zonas de agua abierta y 


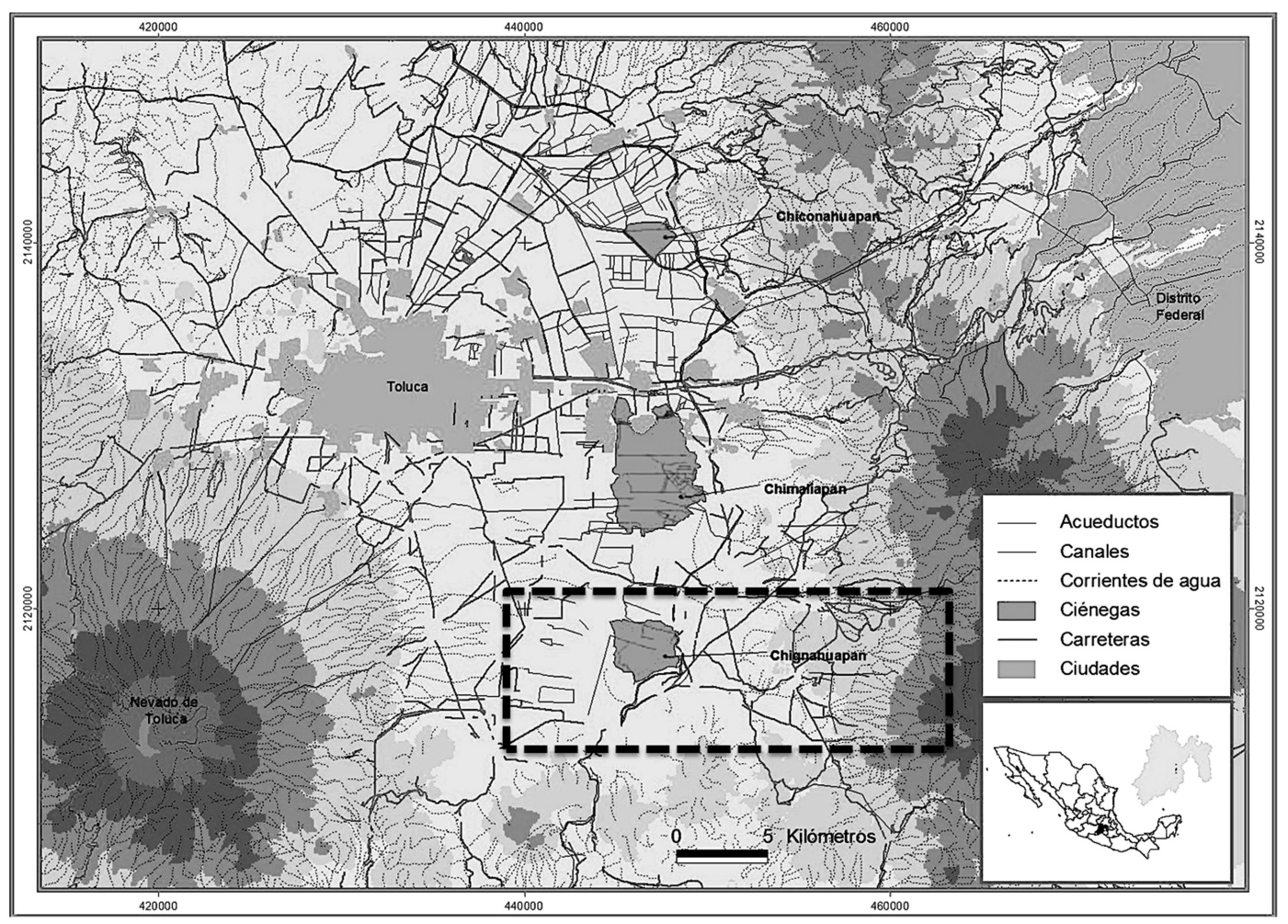

Figura 1. Ubicación de la Ciénega de Chignahuapan.

permanente cuya profundidad máxima es de $2.5 \mathrm{~m}$ y donde prosperan hidrófitas libres sumergidas, de hojas flotantes y libres flotadoras. Rodeando estas zonas, se extienden amplias franjas sometidas a inundación estacional con una profundidad máxima de $1.50 \mathrm{~m}$ y donde la vegetación está constituida principalmente por hidrófitas enraizadas emergentes (Ramos-Ventura, 1999). Las comunidades vegetales presentan especies nativas y exóticas que se mantienen como zonas de alta fragilidad con diferentes grados de perturbación y contaminación (Zepeda-Gómez et al., 2012a). La zona de estudio forma parte de las Ciénegas de Lerma, una región importante en el almacenamiento e infiltración del agua de la cuenca de Lerma y considerada una de las 75 regiones prioritaria, amenazas y con alta biodiversidad (Arriaga-Cabrera et al., 2000). La extensión inundable y de vegetación acuática de las ciénegas y en particular de la de Chignahuapan se han reducido en más de la mitad del área que ocupaban hace un poco más de tres décadas, debido principalmente a la sobreexplotación de los mantos freáticos, el cambio de uso de suelo y la falta de un plan de manejo centrado en los usos locales (Zepeda-Gómez et al., 2012b).

Muestreo. En las zonas estacionalmente inundadas de Chignahuapan se seleccionaron sistemáticamente y en función de la accesibilidad, 24 sitios de muestreo separados por una distancia mínima de $50 \mathrm{~m}$. En febrero de 2011, en cada sitio se colectaron dos muestras de los $10 \mathrm{~cm}$ más superficiales del sedimento (Liu et al., 2005) usando un cilindro de metal de $4.5 \mathrm{~cm}$ de diámetro por $60 \mathrm{~cm}$ de largo (área superficial de $0.00159 \mathrm{~m}^{2}$ ). El área acumulada de muestreo fue de $0.0650 \mathrm{~m}^{2}$. Las muestras se almacenaron en bolsas plásticas y se mantuvieron en un cuarto obscuro a $3{ }^{\circ} \mathrm{C}$ por un mes.

Método se separación de semillas. Una muestra de sedimento de cada sitio se usó para estimar el banco de semillas (BS) por el método de separación de semillas (MSS) para lo cual cada muestra se humedeció y lavó a través de una columna de tamices con diferentes aperturas de mallas (la más pequeña fue de $0.11 \mathrm{~mm}$ ), el material recolectado se secó en un horno de convección marca Yamato (Mod. DKN600) a $20{ }^{\circ} \mathrm{C}$ por $24 \mathrm{~h}$. Posteriormente se extrajeron bajo el microscopio estereoscópico todas las semillas que se encontraron y cada una se colocó por especie en diferentes cajas de plástico para su posterior conteo e identificación. Las especies de semillas extraídas con más de 20 representantes se usaron en una prueba de Tetrazolio (Cloruro de 2,3,5-trifeniltetrazolio), para determinar el porcentaje de semillas viables (Ruíz, 2009).

Método de germinación. La segunda muestra de sedimento colectada de cada sitio se usó para aplicar el método de germinación (MG) según la técnica de van der Valk y Davis (1978). Para ello, las muestras se colocaron en macetas de $15 \mathrm{~cm}$ de diámetro previamente preparadas con una capa de arena estéril y se mantuvieron con luz natural en un inver- 
Tabla 1. Medias \pm error estándar (ES) de los parámetros estimados por el método de separación de semillas (MSS) y el método de germinación (MG). $\mathrm{a}=$ diferencias significativas $(P<0.05) ; \mathrm{b}=$ No hay diferencias significativas $(P>0.05)$.

\begin{tabular}{|c|c|c|c|c|}
\hline \multirow[b]{2}{*}{ Riqueza } & \multirow{2}{*}{$\begin{array}{c}\text { MSS } \\
52\end{array}$} & \multirow{2}{*}{$\begin{array}{c}\text { MG } \\
24\end{array}$} & \multicolumn{2}{|c|}{ Prueba estadística } \\
\hline & & & $t=12.2^{\mathrm{a}}$ & $r^{2}=0.004^{b}$ \\
\hline Especies por muestra & $19 \pm 3$ & $6 \pm 1$ & $z=5.1^{\mathrm{a}}$ & $r^{2}=0.70^{b}$ \\
\hline Densidad (semillas o plántulas m²) & $439,265 \pm 54,358$ & $35,084 \pm 4,431$ & $z=5.1^{\mathrm{a}}$ & $r^{2}=0.044^{b}$ \\
\hline Shannon-Wiener $\left(\mathrm{H}^{\prime}\right)$ & 2.6 & 2.2 & $t=5.2^{\mathrm{a}}$ & $r^{2}=0.008^{b}$ \\
\hline Valor de Pielou $\left(\mathrm{H}^{\prime}{ }_{\max }\right)$ & 4.0 & 3.1 & & \\
\hline Equidad $\left(J^{\prime}\right)$ & 0.67 & 0.71 & & $r^{2}=0.016^{b}$ \\
\hline Dominancia (D) & 0.11 & 0.17 & & \\
\hline Simpson 1/D & 9.0 & 5.8 & & \\
\hline Índice de Morisita & & 0.41 & & \\
\hline Coeficiente de Sørensen & & 0.37 & & \\
\hline
\end{tabular}

nadero a $20-25^{\circ} \mathrm{C}$ por 15 semanas e inundadas con un nivel de agua de 0.5 a $1 \mathrm{~cm}$ por encima de la superficie del suelo. Se revisaron a intervalos de tres días para observar la germinación y crecimiento de las plántulas. Todas las plántulas que emergieron se identificaron, contaron y extrajeron para evitar la competencia entre ellas. Adicionalmente se colocaron cinco macetas con arena estéril, intercaladas con las macetas que contenían las muestras de sedimento, para hacer pruebas de contaminación por semillas locales; durante las pruebas de germinación no se encontraron plántulas en las macetas de control.

Análisis de resultados. La riqueza de especies se consideró como el número de especies presentes en todas las muestras de sedimento utilizadas en cada método (Magurran, 2004; Begon et al., 2006). Para determinar el grado de completitud del inventario obtenido por el MSS y MG, se crearon curvas de acumulación de taxones empleando 2 estimadores no paramétricos: bootstrap y Jacknife 1, los valores se obtuvieron con el programa EstimateS versión 8.2 (Colwell, 2009), empleando 1,000 iteraciones con reemplazo en las muestras. Las comparaciones de las curvas de acumulación de especies están dadas con base en los intervalos de confianza del $95 \%$ generado por el programa EstimateS.

La densidad de semillas y de plántulas se determinó como el número promedio de organismos por muestra de la especie x expresada en metros cuadrados (Begon et al., 2006). La frecuencia y densidad de cada especie obtenida por cada método, se comparó mediante una prueba de Ji cuadrada. La densidad y número de especies por muestra entre los dos métodos de evaluación se comparó con una prueba de U de Mann Withney. Adicionalmente se determinó la diversidad de semillas y plántulas por cada punto de muestreo y la total mediante los índices de diversidad de Shannon-Wiener $\left(\mathrm{H}^{\prime}\right)$ y el inverso del índice de Simpson (Zar, 1999) en el programa PAST 1.90 (Hammer et al., 2001). Los valores obtenidos del índice de Shannon-Wiener en cada método se compararon con una t-Student modificada (Zar, 1999) para establecer diferencias significativas.
Adicionalmente y como complemento de los índices de diversidad, se calculó el índice de uniformidad de Pielou (J') para determinar la equidad en la abundancia de las especies y el valor del índice de Simpson se usó para determinar la dominancia de las mismas (Moreno, 2001). Se usó una prueba de rangos de Sperman para correlacionar las estimaciones del número de especies, densidad, diversidad y valor de Pielou por muestra entre los métodos y para establecer la relación de densidad de las especies comunes a ambas técnicas. La similitud florística entre las técnicas de evaluación se calculó con el coeficiente de similitud de Sørensen y el índice de Morisita (Moreno, 2001). La relación cuantitativa entre la abundancia de las especies por método de evaluación se determinó mediante un Análisis de Correspondencia sin tendencia (DCA) en el programa Past 1.90 (Hammer et al., 2001). Las especies de la vegetación reportadas para la ciénega en Zepeda-Gómez et al. (2012a) se usaron para calcular mediante el coeficiente de similitud de Sørensen, la similitud entre la vegetación, el banco de semillas obtenido por cada método y el total (Moreno, 2001). Los análisis univariados se realizaron con el programa Statistica 7 (StatSoft Inc. 2004). Las clases de plantas acuáticas, subacuáticas y tolerantes se reportan según Lot y Novelo (1978), Lot et al. (1999) y Calderón de Rzedowski y Rzedowski (2001), las formas de vida con base en Sculthorpe (1985) y las malezas de acuerdo con Espinosa-García y Sarukhán (1997) y Calderón de Rzedowski y Rzedowski (2001).

Tabla 2. Riqueza observada y calculada por dos estimadores no paramétricos y porcentaje de eficiencia para cada método de evaluación del banco de semillas MSS = método de separación de semillas; MG = método de germinación.

\begin{tabular}{lccccc}
\hline Método & $\begin{array}{c}\text { Especies } \\
\text { observadas }\end{array}$ & \multicolumn{2}{c}{$\begin{array}{c}\text { Especies } \\
\text { esperadas }\end{array}$} & \multicolumn{2}{c}{$\begin{array}{c}\text { Porcentaje de } \\
\text { eficiencia (\%) }\end{array}$} \\
\hline \multirow{3}{*}{ MSS } & 50 & 65 & 60 & 84 & 92 \\
MG & 24 & 33 & 28 & 74 & 86 \\
\hline
\end{tabular}




\section{Resultados}

Método de separación de semillas (MSS). Se registraron 12,575 semillas de 52 especies diferentes. Esto correspondió a una densidad promedio de 439,265 $\pm 54,358$ semillas por metro cuadrado, con $19 \pm 3$ especies por sitio (Tabla 1).

Los estimadores de riqueza (Tabla 2; Figura 2) indican que el inventario de especies obtenido por este método fluctuó entre $84 \%$ (Jacknife) y $92 \%$ (bootstrap). De las 25 familias de plantas registradas, en 11 de ellas se agrupó cerca del $95 \%$ de la densidad total del BS (Tabla 3), entre ellas sobresalen Cyperaceae (10 especies), Asteraceae y Polygonaceae (5 especies respectivamente) por presentar el mayor número de especies. Los miembros de la familia Cyperaceae agruparon cerca del $26 \%$ de todas las semillas (Tabla $3)$. Asteraceae fue la segunda familia más numerosa (20\% de las semillas), seguida de Apiaceae (16\% de las semillas; Tabla 3). De las especies reconocidas destacan Eleocharis macrostachya, Euphrosyne partheniifolia, Lilaeopsis schaffneriana y Schoenoplectus tabernaemontani (Figura 3, Tabla 4) como las especies con mayor densidad. Las especies más frecuentes fueron Schoenoplectus tabernaemontani, Polygonum punctatum y Urtica dioica (Figura 3).

El $66 \%$ de las semillas extraídas correspondió a plantas acuáticas y subacuáticas, con seis formas de vida de las cuales las hidrófitas enraizadas emergentes fueron las más abundantes (54\%). El $30 \%$ de las semillas pertenecieron a especies terrestres tolerantes y cerca del $50 \%$ a plantas de hábito perenne (Figura 4). La prueba de Tetrazolio mostró en algunos casos bajos porcentajes de viabilidad de las semillas extraídas (Tabla 4).

El $62 \%$ de los sitios de muestreo registraron un valor de diversidad $\left(\mathrm{H}^{\prime}\right)$ entre uno y dos, el restante 37 \% registró

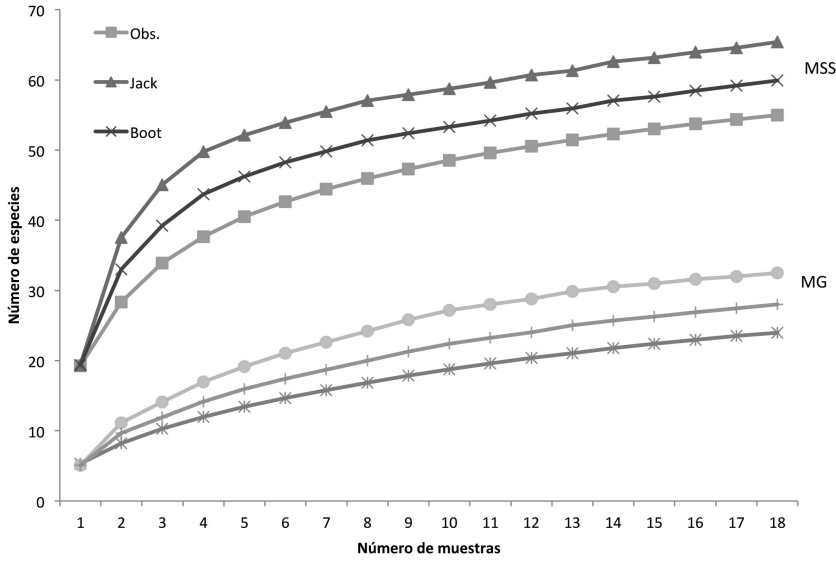

Figura 2. Curvas de acumulación de especies observadas (Obs) y esperadas, utilizando los estimadores no paramétricos de Jacknife (Jack) y bootstrap (Boot), para cada método de evaluación. MSS = método de separación de semillas; $\mathrm{MG}=$ método de germinación.

valores superiores (Tabla 5). De forma general este método indica que el BS tiene una diversidad con tendencia a ser alta $\left(\mathrm{H}^{\prime}\right.$ Total $=2.6$, Tabla 1$)$. La vegetación y el BS obtenido por este método mostraron una baja similitud florística $($ Sørensen $=0.39$ ).

Método de germinación $(M G)$. Por este método se obtuvieron 1,025 individuos de 24 especies. Esto corresponde a una densidad promedio de 35,084 $\pm 4,431$ plántulas por metro cuadrado con $6 \pm 1$ especies por sitio (Tabla 1).

Los estimadores de riqueza (Tabla 2, Figura 2) indican que el inventario de especies fluctuó entre $74 \%$ (Jacknife) y $86 \%$ (bootstrap). Se registraron 14 familias de plántulas, en seis de ellas se agrupó el $77 \%$ de la densidad total. El $24 \%$

Tabla 3. Familias de plantas con mayor densidad promedio de semillas o plántulas por metro cuadrado en el banco de semillas de Chignahuapan. Método de separación de semillas (MSS) y germinación (MG). $\mathrm{n}$ = Número promedio de individuos.

\begin{tabular}{|c|c|c|c|c|c|c|}
\hline \multirow[b]{2}{*}{ FAMILIA } & \multicolumn{3}{|c|}{ MSS } & \multicolumn{3}{|c|}{ MG } \\
\hline & Especies & $n$ & Densidad $\mathrm{sem} / \mathrm{m}^{2}$ & Especies & $\mathbf{n}$ & Densidad plánt $/ \mathrm{m}^{2}$ \\
\hline Apiaceae & 3 & 179 & 112,838 & 2 & 17 & 9,791 \\
\hline Asteraceae & 5 & 226 & 141,892 & & & \\
\hline Callitrichaceae & & & & 1 & 26 & 16,348 \\
\hline Caryophyllaceae & 4 & 42 & 26,408 & & & \\
\hline Cruciferae & 2 & 84 & 53,127 & 1 & 20 & 12,408 \\
\hline Cyperaceae & 10 & 297 & 189,942 & 4 & 40 & 25,014 \\
\hline Haloragaceae & 1 & 67 & 41,842 & & & \\
\hline Lemnaceae & 1 & 15 & 14,671 & & & \\
\hline Poaceae & 3 & 13 & 8,371 & 1 & 1 & 628 \\
\hline Polygonaceae & 5 & 49 & 37,745 & & & \\
\hline Ranunculaceae & & & & 1 & 28 & 17,606 \\
\hline Scrophulariaceae & 1 & 70 & 44,223 & & & \\
\hline Urticaceae & 1 & 24 & 15,012 & & & \\
\hline Total & 36 & 1,066 & 670,304 & 10 & 132 & 81,795 \\
\hline Total General & 52 & 1,122 & 705,200 & 24 & 169 & 106,265 \\
\hline
\end{tabular}


Tabla 4. Familia, especie, categoría, forma de vida, frecuencia, número de individuos y densidad promedio por metro cuadrado de las especies por el método de separación de semillas (MSS) y germinación (MG).

\begin{tabular}{|c|c|c|c|c|c|c|c|c|c|c|c|}
\hline Familia & Especie & Tipo & Categoría & $\begin{array}{l}\text { Forma } \\
\text { de vida }\end{array}$ & $\mathbf{n}$ & $\begin{array}{c}\text { MSS } \\
\text { Densidad } \\
\text { Sem. } / \mathrm{m}^{2}\end{array}$ & $\begin{array}{c}\text { Frecuencia } \\
\%\end{array}$ & $\begin{array}{c}\text { Viabilidad } \\
\%\end{array}$ & $\mathbf{n}$ & $\begin{array}{c}\text { MG } \\
\text { Densidad } \\
\text { Plánt. } / \mathrm{m}^{2}\end{array}$ & $\begin{array}{c}\text { Frecuencia } \\
\%\end{array}$ \\
\hline Amaranthaceae & Amaranthus hybridus $\mathrm{L}$. & An & $\mathrm{T}$ & $\mathrm{m}$ & 7 & 4,491 & 39 & 10 & & & \\
\hline Apiaceae & $\begin{array}{l}\text { Hydrocotyle } \\
\text { ranunculoides L. }\end{array}$ & $\mathrm{Pe}$ & A & hee & 9 & 5,423 & 44 & 50 & & & \\
\hline Apiaceae & $\begin{array}{l}\text { Hydrocotyle verticillata } \\
\text { Thunb. }\end{array}$ & $\mathrm{Pe}$ & A & hee & 15 & $9,641 a$ & $17 \mathrm{a}$ & 45 & 1 & $629 b$ & $11 \mathrm{a}$ \\
\hline Apiaceae & $\begin{array}{l}\text { Lilaeopsis schaffneriana } \\
\text { (Schltdl.) Coult. y Rose }\end{array}$ & PeAn & A & hee & 156 & $97,774 a$ & $44 a$ & 62 & 16 & $9,791 b$ & $11 b$ \\
\hline Asteraceae & Aster subulatus Michx & An & $\mathrm{T}$ & $\mathrm{m}$ & & & & & 2 & 1,258 & 17 \\
\hline Asteraceae & $\begin{array}{l}\text { *Euphrosyne partheniifolia } \\
\text { DC. }\end{array}$ & PeAn & $\mathrm{S}$ & hee & 216 & $136,024 a$ & $83 a$ & 32 & 6 & $3,720 \mathrm{~b}$ & $6 b$ \\
\hline Asteraceae & Gnaphalium luteo-album L. & An & $\mathrm{T}$ & $\mathrm{m}$ & & & & & 4 & 2,515 & 6 \\
\hline Asteraceae & Gnaphalium sp. & An & $\mathrm{T}$ & $\mathrm{m}$ & 2 & 1,258 & 6 & & & & \\
\hline Asteraceae & $\begin{array}{l}\text { *Jaegeria bellidiflora } \\
\text { (Sessé \& Moc. ex DC.) } \\
\text { Torres y Beaman }\end{array}$ & PeAn & A & hee & 1 & $629 a$ & $6 a$ & & 2 & $943 b$ & $33 b$ \\
\hline Asteraceae & $\begin{array}{l}\text { *Jaegeria glabra (S. } \\
\text { Watson) B.L.Rob. }\end{array}$ & An & A & hee & 3 & 1,572 & 11 & 10 & & & \\
\hline Asteraceae & $\begin{array}{l}\text { Melampodium } \\
\text { bibracteatum S. Watson }\end{array}$ & An & $S$ & hee & 2 & 1,153 & 33 & 60 & & & \\
\hline Callitricaceae & $\begin{array}{l}\text { Callitriche deflexa A. } \\
\text { Braun ex Hegelm. }\end{array}$ & An & A & hee & & & & & 26 & 16,348 & 67 \\
\hline Caryophyllaceae & Arenaria bourgaei Hemsl. & $\mathrm{Pe}$ & $S$ & hetp & 29 & $18,486 a$ & $56 a$ & & 4 & $2,305 b$ & $17 \mathrm{~b}$ \\
\hline Caryophyllaceae & Arenaria paludicola B.L.Rob. & $\mathrm{Pe}$ & $\mathrm{S}$ & hee & 5 & $2,892 \mathrm{a}$ & $28 \mathrm{a}$ & & 5 & $2,934 a$ & $83 b$ \\
\hline Caryophyllaceae & $\begin{array}{l}\text { Drymaria glandulosa } \\
\text { Bartling }\end{array}$ & An & T & & 7 & 4,401 & 11 & & & & \\
\hline Caryophyllaceae & $\begin{array}{l}\text { Drymaria villosa Chan. } \\
\text { \& Schltdl. }\end{array}$ & An & $\mathrm{T}$ & $\mathrm{m}$ & 1 & 629 & 6 & & & & \\
\hline Chenopodiaceae & Chenopodium sp. & An & $\mathrm{T}$ & $\mathrm{m}$ & 5 & 2,829 & 11 & & & & \\
\hline Crasulaceae & Tillaea saginoides Maxim. & An & $\mathrm{S}$ & hee & & & & & 3 & 1,886 & 39 \\
\hline Cruciferae & $\begin{array}{l}\text { Rorippa mexicana (Moc. } \\
\text { \& Sessé) Standl. }\end{array}$ & An & S & hee & 53 & 33,235 & 39 & & & & \\
\hline Cruciferae & $\begin{array}{l}\text { Rorippa pinnata (Moc. } \\
\text { \& Sessé) Rollins }\end{array}$ & An & S & hee & 32 & $19,892 a$ & $61 \mathrm{a}$ & & 20 & $12,408 b$ & $11 b$ \\
\hline Cyperaceae & Carex sp. & & $S$ & hee & 7 & 4,559 & 28 & & & & \\
\hline Cyperaceae & Cyperus sp. & & & & 1 & 629 & 6 & & & & \\
\hline Cyperaceae & $\begin{array}{l}\text { Eleocharis acicularis (L.) } \\
\text { Roem. \& Schilt. }\end{array}$ & PeAn & A & hee & & & & & 14 & 8,860 & 6 \\
\hline Cyperaceae & Eleocharis bonariensis Nees & $\mathrm{Pe}$ & A & hee & 28 & 17,354 & 83 & 30 & & & \\
\hline Cyperaceae & Eleocharis densa Benth. & $\mathrm{Pe}$ & $\mathrm{S}$ & hee & 24 & 14,776 & 56 & 15 & & & \\
\hline Cyperaceae & Eleocharis dombeyana Kunth & $\mathrm{Pe}$ & $\mathrm{S}$ & hee & 50 & $31,543 a$ & $67 a$ & & 10 & $6,094 b$ & $6 b$ \\
\hline Cyperaceae & $\begin{array}{l}\text { Eleocharis macrostachya } \\
\text { Britton }\end{array}$ & $\mathrm{Pe}$ & $S$ & hee & 87 & $54,568 a$ & $78 \mathrm{a}$ & 88 & 12 & $7,545 b$ & $6 b$ \\
\hline Cyperaceae & $\begin{array}{l}\text { Eleocharis montana (Kunth) } \\
\text { Roem. \& Schult. }\end{array}$ & $\mathrm{Pe}$ & $S$ & hee & 4 & 2,578 & 56 & & & & \\
\hline Cyperaceae & $\begin{array}{l}+ \text { Schoenoplectus } \\
\text { americanus (Pers.) Volkart } \\
\text { ex Schinz \& Keller }\end{array}$ & $\mathrm{Pe}$ & A & hee & 1 & 629 & 6 & 10 & & & \\
\hline Cyperaceae & $\begin{array}{l}\text { +Schoenoplectus californicus } \\
\text { (C.A. Meyer) Soják }\end{array}$ & $\mathrm{Pe}$ & A & hee & 5 & 3,249 & 33 & 30 & & & \\
\hline Cyperaceae & $\begin{array}{l}\text { Schoenoplectus } \\
\text { tabernaemontani (C.C. } \\
\text { Gmel.) Palla }\end{array}$ & $\mathrm{Pe}$ & A & hee & 91 & $57,323 a$ & $100 \mathrm{a}$ & 28 & 4 & $2,515 b$ & $11 b$ \\
\hline Euphorbiaceae & Euphorbia nutans Lag. & & $\mathrm{T}$ & $\mathrm{m}$ & 1 & 629 & 6 & & & & \\
\hline
\end{tabular}


Tabla 4. Continuación

\begin{tabular}{|c|c|c|c|c|c|c|c|c|c|c|c|}
\hline Familia & Especie & Tipo & Categoría & $\begin{array}{l}\text { Forma } \\
\text { de vida }\end{array}$ & $\mathbf{n}$ & $\begin{array}{c}\text { MSS } \\
\text { Densidad } \\
\text { Sem. } / \mathrm{m}^{2}\end{array}$ & $\begin{array}{l}\text { Frecuencia } \\
\quad \%\end{array}$ & $\begin{array}{l}\text { Viabilidad } \\
\quad \%\end{array}$ & $\mathbf{n}$ & $\begin{array}{c}\text { MG } \\
\text { Densidad } \\
\text { Plánt./m² }\end{array}$ & $\begin{array}{c}\text { Frecuencia } \\
\%\end{array}$ \\
\hline Haloragaceae $^{a}$ & Myriophyllum sp. & $\mathrm{Pe}$ & A & hes & 67 & 41,842 & 61 & & & & \\
\hline Isoetaceae ${ }^{\mathrm{a}}$ & Isoetes sp. & & A & hee & 6 & 3,773 & 39 & & & & \\
\hline Lemnaceae & Lemna sp. & An & A & hlf & 15 & 9,432 & 22 & & & & \\
\hline Malvaceae & Malva parviflora L. & An & $\mathrm{T}$ & $\mathrm{m}$ & 1 & 629 & 11 & & & & \\
\hline Najadaceae $^{a}$ & $\begin{array}{l}\text { +Najas guadalupensis } \\
\text { (Spreng.) Magnus }\end{array}$ & An & A & hes & 2 & 1,100 & 22 & 30 & & & \\
\hline Menyanthaceae $^{\mathrm{a}}$ & $\begin{array}{l}\text { +Nymphoides fallax } \\
\text { Ornduff }\end{array}$ & PeAn & A & hehf & 4 & 2,641 & 56 & 10 & & & \\
\hline Onagraceae & Epilobium ciliatum Raf. & $\mathrm{Pe}$ & S & hee & 2 & $1,258 a$ & $11 \mathrm{a}$ & & 1 & $629 b$ & $11 \mathrm{a}$ \\
\hline Otras & Otras (2) & & & & 19 & 11,695 & 6 & & & & \\
\hline Oxalidaceae & Oxalis corniculata L. & PeAn & $\mathrm{T}$ & $\mathrm{m}$ & 2 & $1,258 a$ & $6 a$ & & & & \\
\hline Poaceae & $\begin{array}{l}\text { Echinochloa holciformis } \\
\text { (Kunth) Chase }\end{array}$ & An & S & hee & 8 & 4,779 & 56 & & & & \\
\hline Poaceae & $\begin{array}{l}\text { Echinochloa oplismenoides } \\
\text { (Kunth) Chase }\end{array}$ & An & S & hee & 4 & 2,335 & 39 & & & & \\
\hline Poaceae & Eleusine indica L. (Gaertn.) & An & $\mathrm{T}$ & & 2 & 1,258 & 17 & & & & \\
\hline Poaceae & Pasto & & & & & & & & 1 & 629 & 61 \\
\hline Polygonaceae & $\begin{array}{l}\text { +Polygonum } \\
\text { hydropiperoides Michx. }\end{array}$ & An & S & hee & 2 & 943 & 11 & 30 & & & \\
\hline Polygonaceae & +Polygonum lapathifolium L. & An & S & hee & 13 & $8,174 a$ & $72 a$ & 54 & 1 & $629 b$ & $6 b$ \\
\hline Polygonaceae & $\begin{array}{l}\text { Polygonum mexicanum } \\
\text { Small }\end{array}$ & An & S & hee & 2 & 1,258 & 11 & 33 & & & \\
\hline Polygonaceae & $\begin{array}{l}+ \text { Polygonum punctatum } \\
\text { Elliott }\end{array}$ & An & S & hee & 11 & $6,956 a$ & $89 a$ & 75 & 2 & $943 b$ & $6 b$ \\
\hline Polygonaceae & Rumex flexicaulis Riechb. F. & An & $\mathrm{T}$ & $\mathrm{m}$ & & & & & 1 & 629 & 6 \\
\hline Polygonaceae & Rumex sp. & An & $\mathrm{T}$ & $\mathrm{m}$ & 21 & 13,249 & 56 & 55 & & & \\
\hline $\begin{array}{l}\text { Potamogeto- } \\
\text { naceae }^{\mathrm{a}}\end{array}$ & Potamogeton sp. & $\mathrm{Pe}$ & A & hes & 2 & 978 & 50 & & & & \\
\hline Ranunculaceae & $\begin{array}{l}\text { Ranunculus cymbalaria } \\
\text { Pursh }\end{array}$ & $\mathrm{Pe}$ & S & hee & & & & & 28 & 17,606 & 22 \\
\hline Ranunculaceae & $\begin{array}{l}\text { Ranunculus dichotomus } \\
\text { Moc. y Sessé }\end{array}$ & $\mathrm{Pe}$ & $\mathrm{T}$ & & 1 & 629 & 6 & & & & \\
\hline Scrophulariaceae & +Limosella aquatica L. & $\mathrm{Pe}$ & A & hee & 70 & $44,223 a$ & $33 a$ & & 6 & $3,563 b$ & $72 b$ \\
\hline Solanaceae & $\begin{array}{l}\text { Jaltomata procumbens } \\
\text { (Cav.) J.L. Gentry }\end{array}$ & An & $\mathrm{T}$ & $\mathrm{m}$ & 3 & 1,729 & 22 & 10 & & & \\
\hline Solanaceae & Solanum rostratum Dunal. & An & $\mathrm{T}$ & $\mathrm{m}$ & 2 & 1,258 & 6 & 5 & & & \\
\hline Typhaceae & +Typha latifolia L. & $\mathrm{Pe}$ & A & hee & & & & & 1 & 629 & 6 \\
\hline Urticaceae & Urtica dioica L. & $\mathrm{Pe}$ & $\mathrm{T}$ & $\mathrm{m}$ & 24 & $15,012 \mathrm{a}$ & 89a & & 2 & $1,258 b$ & $6 b$ \\
\hline $\begin{array}{l}\text { Verbenaceae } \\
\text { Total } 28\end{array}$ & $\begin{array}{l}\text { Verbena bipinnatifida Nutt. } \\
\mathbf{6 0}\end{array}$ & An & $\mathrm{T}$ & $\mathrm{m}$ & 1 & 629 & 6 & & & & \\
\hline
\end{tabular}

$\mathrm{n}$ = número de individuos promedio; sem. = semillas; plán. = plántulas. An = anual, Pe = perenne, $\mathrm{A}=$ planta acuática, $\mathrm{S}=$ subacuáticas, $\mathrm{T}=$ tolerantes, hee = hidrófita enraizada emergente, hehf = hidrófita enraizada de hojas flotantes, hes = hidrófita enraizada sumergida, hetp = hidrófita enraizada de tallos postrados, hls = hidrófita libre sumergida, hlf = hidrófita libre flotadora, m = maleza, @ = familias de plantas estrictamente acuáticas, ${ }^{*}=$ especies endémicas de México, + = especies invasoras. Diferentes superíndices indican diferencias significativas entre la frecuencia y densidad obtenida para cada especie por cada método y usando una prueba de Ji-cuadrada con una significancia de $\mathrm{P}<0.05$.

de las plántulas se agrupó en cuatro especies de la familia Cyperaceae de las que Schoenoplectus tabernaemontani fue la más abundante. La segunda familia con mayor densidad fue Ranunculaceae (Tabla 3) y el $17 \%$ de las plántulas evaluadas provinieron únicamente de Ranunculus cymbalaria. Junto con esta última especie Callitriche deflexa y Rorippa pinnata destacan por ser las plantas con mayor densidad de todo el BS (Figura 3). Arenaria paludícola, Limosella aquatica y Callitriche deflexa fueron las más frecuentes (Figura 3, Tabla 4).

Las semillas que germinaron correspondieron principalmente a especies perennes $(63 \%)$ y cerca del $40 \%$ a plantas con crecimiento anual. El $34 \%$ fueron plantas acuáticas y el $47 \%$ subacuáticas, solo se registraron hidrófitas enraizadas 

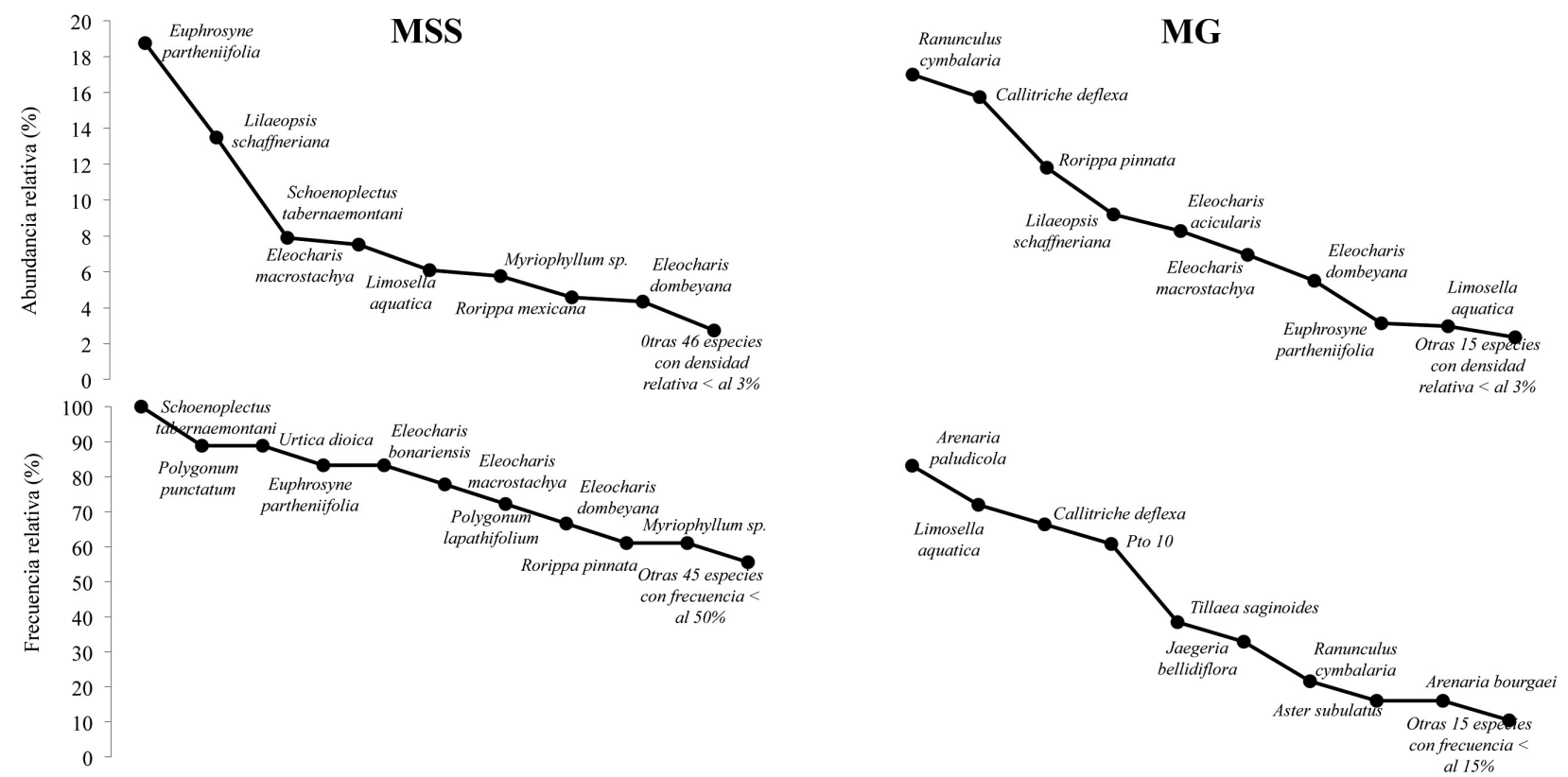

Figura 3. Graficas de rango-abundancia relativa y rango-frecuencia relativa de las especies por método de evaluación. MSS = método de separación de semillas y MG = método de germinación.

emergentes $(78 \%)$ y de tallos postrados (4\%). El $17 \%$ de las germinaciones correspondió a plantas terrestres tolerantes (Figura 4).

La diversidad del $70 \%$ de los sitios de muestreo se ubicó en el rango de $\mathrm{H}^{\prime}=1.0$ y 2.0, el $30 \%$ restante registro valores inferiores (Tabla 5). De forma general este método predijo un BS con diversidad intermedia $\left(\mathrm{H}^{\prime}{ }_{\text {Total }}=2.2\right.$, Tabla 2). La vegetación y el BS obtenido por este método mostraron una similitud florística intermedia $($ Sørensen $=0.56)$.

Comparación de métodos. El número total de especies obtenida por cada método difirió significativamente $(P<0.05$; Tabla 1), a través del MSS se obtienen más del doble de especies que con el MG, por lo que su eficiencia en la determinación de la riqueza es superior (Tabla 1,2). Con el MSS se obtienen tres veces más especies por muestra y diez veces más organismos $(P<0.05$; Tabla 1$)$ que por el MG. Además, el MSS permite visualizar una comunidad más diversa aunque con una equidad menor (Tabla 1). Las correlaciones por rangos entre las estimaciones obtenidas por cada método fueron muy pobres (Tabla 1).

Conjugando los registros de especies obtenidos por ambos métodos, el BS de los sedimentos de la ciénega se compone de 60 especies (Tabla 4), de las cuales $60 \%$ (36 especies) se registraron únicamente en MSS y $15 \%$ (9 especies) exclusivamente en MG. De las 15 especies comunes a ambos métodos, 14 difirieron significativamente en su abundancia y solo Jaegeria bellidiflora fue la especie más densa en MG que en MSS (Tabla 4). La frecuencia de 13 especies también difirió entre las técnicas empleadas, de las 15 especies compartidas solo dos fueron significativamente más frecuentes en MG que en MSS (Tabla 4). Solo Limosella aquatica $(r=$ $0.5 ; P=0.02)$, Hydrocotyle verticillata $(r=0.9 ; P=0.001)$ y Arenaria bourgaei $(r=0.5 ; P=0.05)$, mostraron una rela-
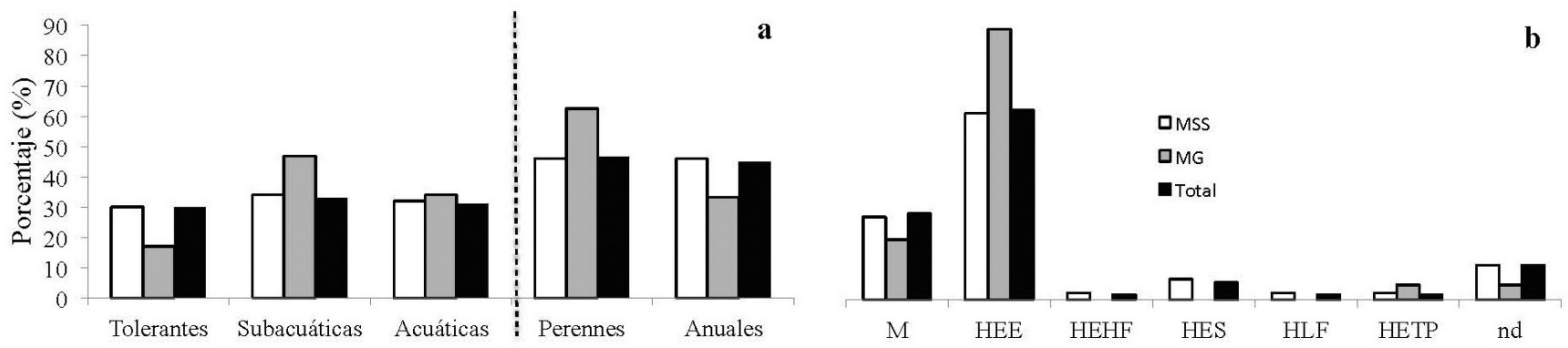

Figura 4. a) Porcentajes de categorías y b) formas de vida del banco de semillas de Chignahuapan registradas por el método de separación de semillas (MSS) y el de germinación (MG). Formas de vida: HEE = hidrófila enraizada emergente; HEHF = hidrófita enraizada de hojas flotantes; HES = hidrófila enraizada sumergida; HETP = hidrófita enraizada de tallos postrados; HLF = hidrófita libre flotadora; HEHS = hidrófita enraizada de hojas sumergidas, nd = no determinadas. 
Tabla 5. Valores de diversidad para cada sitio de muestreo y método. MSS = método de separación de semillas, MG = método de germinación.

\begin{tabular}{|c|c|c|c|c|c|c|c|c|}
\hline \multirow[t]{2}{*}{ Sitio } & \multicolumn{2}{|c|}{ Shannon-Wiener $\left(\mathrm{H}^{\prime}\right)$} & \multicolumn{2}{|c|}{ Valor de Pielou $\left(\mathrm{H}_{\max }^{\prime}\right)$} & \multicolumn{2}{|c|}{ Equidad (J') } & \multicolumn{2}{|c|}{ Dominancia (D) } \\
\hline & MSS & MG & MSS & MG & MSS & MG & MSS & MG \\
\hline 1 & 1.7 & 2.2 & 2.1 & 3.2 & 0.8 & 0.7 & 0.2 & 0.2 \\
\hline 2 & 1.7 & 2.2 & 2.1 & 2.9 & 0.8 & 0.7 & 0.2 & 0.2 \\
\hline 3 & 1.7 & 1.4 & 1.9 & 2.9 & 0.9 & 0.5 & 0.2 & 0.4 \\
\hline 4 & 1.1 & 2.4 & 1.4 & 3.1 & 0.8 & 0.8 & 0.4 & 0.1 \\
\hline 5 & 1.3 & 2.0 & 1.8 & 3.1 & 0.7 & 0.6 & 0.3 & 0.2 \\
\hline 6 & 1.3 & 2.3 & 1.8 & 3.1 & 0.7 & 0.7 & 0.3 & 0.1 \\
\hline 7 & 1.0 & 1.9 & 1.4 & 3.1 & 0.7 & 0.6 & 0.4 & 0.2 \\
\hline 8 & 1.6 & 1.5 & 1.8 & 2.2 & 0.9 & 0.7 & 0.2 & 0.3 \\
\hline 9 & 0.5 & 1.8 & 1.4 & 2.9 & 0.4 & 0.6 & 0.7 & 0.2 \\
\hline 10 & 1.0 & 1.6 & 1.4 & 2.9 & 0.8 & 0.5 & 0.4 & 0.3 \\
\hline 11 & 1.3 & 1.6 & 2.2 & 2.9 & 0.6 & 0.6 & 0.5 & 0.3 \\
\hline 12 & 1.0 & 2.0 & 1.4 & 2.9 & 0.7 & 0.7 & 0.4 & 0.2 \\
\hline 13 & 1.6 & 1.4 & 2.1 & 2.6 & 0.7 & 0.5 & 0.3 & 0.3 \\
\hline 14 & 0.9 & 1.7 & 1.4 & 2.8 & 0.6 & 0.6 & 0.5 & 0.2 \\
\hline 15 & 0.6 & 1.9 & 0.7 & 3.0 & 0.9 & 0.6 & 0.5 & 0.2 \\
\hline 16 & 1.7 & 2.2 & 2.1 & 3.2 & 0.8 & 0.7 & 0.2 & 0.2 \\
\hline 17 & 0.9 & 2.4 & 1.4 & 3.0 & 0.7 & 0.8 & 0.5 & 0.1 \\
\hline 18 & 0.6 & 1.9 & 0.7 & 3.0 & 0.9 & 0.6 & 0.5 & 0.2 \\
\hline 19 & 1.1 & 2.1 & 1.4 & 3.0 & 0.8 & 0.7 & 0.4 & 0.2 \\
\hline 20 & 0.5 & 1.1 & 1.1 & 2.9 & 0.4 & 0.4 & 0.7 & 0.5 \\
\hline 21 & 1.7 & 2.2 & 2.1 & 2.9 & 0.8 & 0.7 & 0.2 & 0.2 \\
\hline 22 & 1.0 & 1.9 & 1.4 & 3.1 & 0.7 & 0.6 & 0.4 & 0.2 \\
\hline 23 & 1.6 & 1.5 & 1.8 & 2.2 & 0.9 & 0.7 & 0.2 & 0.3 \\
\hline 24 & 0.5 & 1.8 & 1.4 & 2.9 & 0.4 & 0.6 & 0.7 & 0.2 \\
\hline Prom. & 1.2 & 1.9 & 1.6 & 2.9 & 0.7 & 0.6 & 0.4 & 0.2 \\
\hline D.E. & 0.4 & 0.3 & 0.4 & 0.3 & 0.2 & 0.1 & 0.2 & 0.1 \\
\hline C.V. & 36.0 & 18.2 & 26.7 & 8.8 & 21.0 & 14.6 & 39.1 & 39.8 \\
\hline
\end{tabular}

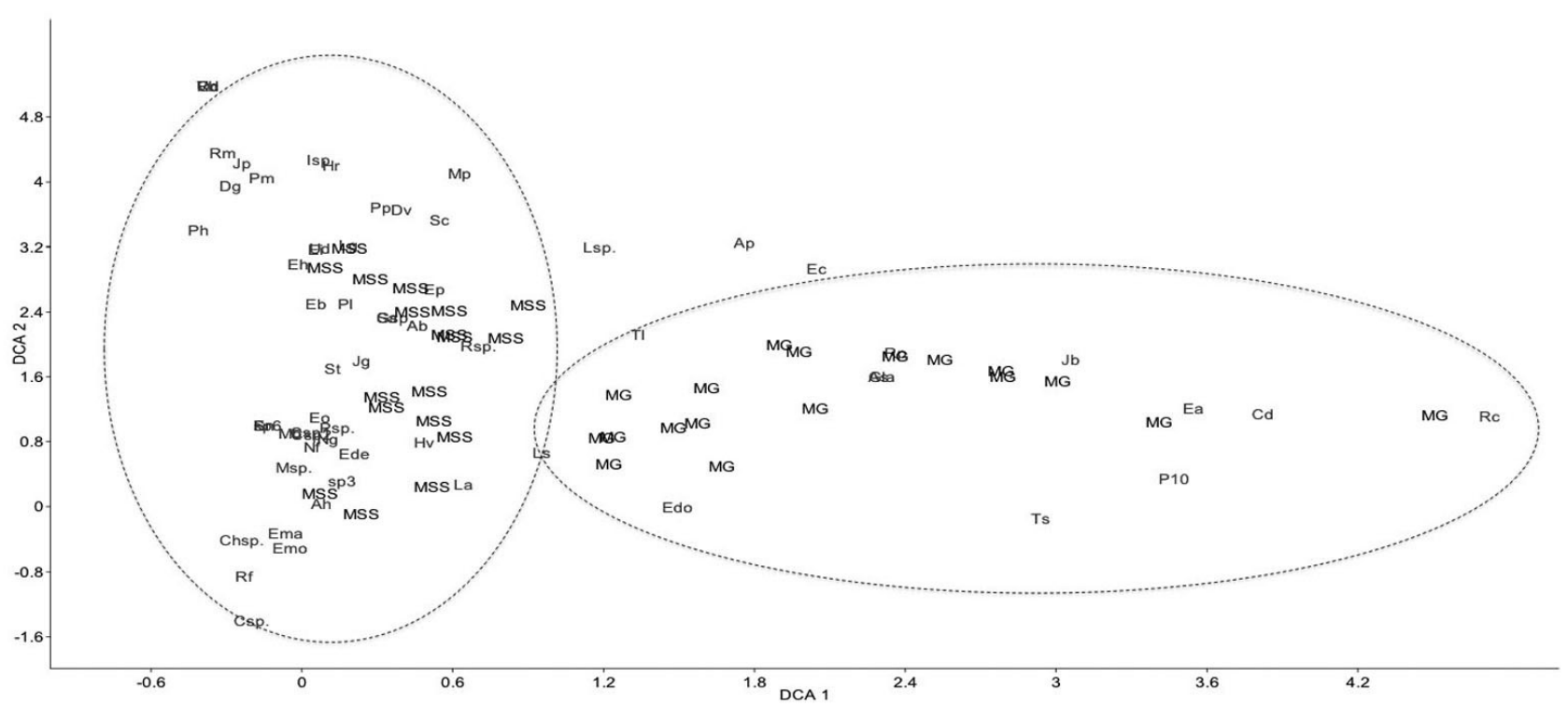

Figura 5. Análisis de correspondencia sin tendencia (DCA) del banco de semillas obtenido por el método de separación de semillas (MSS) y el de germinación (MG). Para las iniciales de las especies ver Cuadro 4. Los eigenvalues de los ejes 1 y 2 son 0.80 y 0.19 respectivamente. 
ción positiva y significativa entre la densidad de semillas y de plántulas.

La similitud florística entre ambos métodos fue relativamente baja e inferior al $45 \%$ (Tabla 1). Las diferencias en las comunidades obtenidas por cada técnica se observan en el diagrama de ordenación DCA (Figura 5). El primer eje separa completamente los dos métodos empleados, la mayor dispersión en la ordenación del MSS indica una mayor variabilidad en las estimaciones del BS. El valor de la raíz 1 $(r=0.80)$, característica para el primer eje, indica una correlación alta entre los métodos de evaluación y la abundancia de las especies.

Considerando las especies obtenidas por ambos métodos, el BS total presentó una baja similitud con la vegetación establecida $($ Sørensen $=0.43$ ).

\section{Discusión}

Los métodos probados muestran diferencias importantes, tanto en la determinación de la composición de especies del banco como en la abundancia de las mismas (Tablas 1, 4). Se encontró que MSS predijo una riqueza, diversidad y densidad, significativamente mayor que MG (Tabla 1), una tendencia que se ha documentado en otras investigaciones (Brown, 1992; Ishikawa-Goto y Tsuyuzaki, 2004; Piudo y Cavero, 2005; Espeland, 2010) y que de acuerdo con las curvas de acumulación de especies (Figura 2) y los reportes de otros autores (Espeland, 2010), podría apoyar la idea de que con menos muestras se puede tener una representación confiable de la composición del banco.

La alta densidad de semillas detectada por MSS respecto a MG (Tabla 1), se debe a que MSS, al no estar influenciado por los requerimientos de germinación de las diferentes especies, detecta tanto las semillas potencialmente germinables como las que están en estado latente y las que no son viables (Piudo y Cavero, 2005). Contar todas las semillas de un volumen de suelo es la forma más precisa de considerar la mayor cantidad semillas y de especies de un lugar (Mesgaran et al., 2007). Sin embargo, al incluir elementos que nunca germinarán, se sobreestima el BS, cuyo valor reside en los propágulos vivos y viables, que al germinar contribuirán a mantener las poblaciones de plantas adultas. Para resolver este inconveniente y conocer la fracción de semillas viables, es necesario invertir más tiempo para hacer pruebas de viabilidad después de aplicar MSS (Fenner y Thompson, 2005). La prueba de Tetrazolio aplicada demostró que no todas las semillas extraídas bajo el microscopio son viables y explica que en los resultados se observen significativamente más especies por muestra y más de diez veces más semi1 las $/ \mathrm{m}^{2}$ por MSS que por MG (Tabla 1). Además indicó que MG no reflejó la abundancia absoluta de semillas del suelo, ya que no fue eficiente en detectar 14 especies de plantas con semillas viables y porque, en las semillas registradas por ambos métodos, las tasas de germinación esperadas son superiores al número de plántulas $/ \mathrm{m}^{2}$ determinadas por MG (Tabla 4). MSS permitió ubicar especies adicionales a las localizadas por MG como Eleocharis bonariensis, E. densa, Jaegeria glabra, Najas guadalupensis, Nymphoides fallax, Schoenoplectus americanus y S. californicus, entre otras (Tabla 4), algunas de las cuales no se registran en la vegetación, pero cuyos valores de viabilidad y densidad de sus semillas sugieren ciertas posibilidades de regeneración a partir del BS.

Con MG se cuentan las germinaciones exitosas, de tal forma que los resultados reflejan la fracción del banco potencialmente activo (Bernhardt et al., 2008). El uso de este método está más generalizado porque teóricamente es menos laborioso que MSS (Kalamees y Zobel, 2002; Clarke y Dorji, 2008; Espeland, 2010). Sin embargo, una de sus principales restricciones es su ineficiencia al determinar el BS de muestras multiespecíficas (Ishikawa-Goto y Tsuyuzaki, 2004), como las de la zona de estudio, en la cual se mezclan varias especies de acuáticas, subacuáticas y tolerantes (Figura 4), cada una con requerimientos de germinación particulares. Con MG los sedimentos se colocan bajo condiciones ambientales constantes y los niveles óptimos a los que se deben instalar son difíciles de definir, porque los factores precisos para la germinación de las diferentes especies acuáticas y tolerantes no están completamente documentados. Las especies pueden variar en la cantidad de agua, luz y temperatura que requieren para germinar (Baskin y Baskin, 2001), de tal forma que al mantener homogéneas las condiciones de germinación en el invernadero, se subestima el BS, ya que habrá una fracción desconocida que no se evaluará dado que no tuvo las condiciones para germinar o porque no tuvo los estímulos necesarios para romper la latencia (Cronk y Fennessy, 2001). Estas limitaciones, propias de MG, explican el menor valor de diversidad y de formas de vida que se registró por este método, así como las diferencias de densidad y frecuencia de las especies comunes a ambas técnicas (Tablas 1,4), el menor número de especies tolerantes y la ausencia de más de 30 especies que no germinaron, pero que si se registraron en MSS, 14 de ellas con algún porcentaje de semillas viables (Tabla 4).

Los bajos valores de similitud encontrados (Tabla 1, Figura 5) y la inexistencia de relación entre las especies de ambos métodos muestran que cada técnica tiene un poder predictivo diferente, lo cual condiciona las inferencias derivadas de ellas. El MSS evidenció una riqueza y abundancia de semilla alta, mientras que MG, si bien no fue eficiente es estimar la riqueza y tampoco reflejar la abundancia total de semillas viables, si mostró una similitud más alta con la vegetación establecida y predijo que especies emergerán con mayor facilidad del banco. Los resultados apoyan las conclusiones de Ishkawa-Goto y Tsuyuzaki (2004) y Espeland et al. (2010) que recomiendan la aplicación de más de una técnica para tener un panorama más amplio y no sesgado de la composición y estructura del banco de un área. Aunado a 
lo anterior y para superar las deficiencias metodológicas de MG, es necesario realizar más investigaciones que determinen las condiciones óptimas de germinación y de establecimiento de las plántulas de acuáticas y subacuáticas, tanto en invernadero como en campo.

En esta investigación la aplicación simultánea de los dos métodos estándar, permitió detectar 60 especies de plantas vasculares en el BS de Chignahuapan (Tabla 1), valor que es ligeramente superior al intervalo citado por Cronk y Fennessy (2001) para humedales de zonas templadas (1 a 59 especies), el mayor porcentaje corresponde a especies subacuáticas (33\%) e hidrófitas enraizadas emergentes (55\%; Figura 4), que son precisamente la categoría y la forma de vida de las plantas características de los humedales con inundación estacional y en particular de la vegetación que actualmente prospera en Chignahuapan (Ramos-Ventura, 1999; Zepeda-Gómez et al., 2012a). El $30 \%$ de todas las especies del BS tienen representantes en la vegetación establecida del humedal (Tabla 3), lo cual podría asegurar la permanencia de casi el $80 \%$ de las plantas que actualmente se observan en la vegetación de la zona de estudio. No obstante, la baja similitud entre la vegetación establecida y el BS puede estar asociado a las estrategias reproductivas y a los patrones del ciclo de vida de las diferentes especies y puede sugerir condiciones inapropiadas para la germinación y establecimiento de las mismas (Lu et al., 2010). Cerca de 20 especies que no están presentes en la vegetación podrían incorporarse a ella a partir del BS si las condiciones lo permitieran. Entre ellas sobresalen algunas hidrófitas que en la zona de estudio tienen una distribución limitada (RamosVentura, 1999), aunque son abundantes en otras zonas inundadas de México, como Najas guadalupensis, Potamogeton sp., Schoenoplectus californicus y S. americanus, así como especies con amplia tolerancia a los disturbios antrópicos como Hydrocotyle ranunculoides, Jaegeria bellidiflora, Lilaeopsis schaffneriana, Myriophyllum sp., Nymphoides fallax, Rorippa pinnata, Typha latifolia y varias especies de Eleocharis.

La zona de muestreo está sometida a inundación estacional y como área de transición, sus bancos son una fuente importante de especies malezoides y no autóctonas (Leck, 2003; Alexander y D'Antonio, 2003). En las muestras analizadas se ubicaron 15 especies de plantas tolerantes ( $25 \%)$, la mayoría oportunistas terrestres que se han adaptado a sitios estacionalmente inundados como es el caso de Amaranthus hybridus, Drymaria spp., Rumex spp. y Urtica dioica, entre otras. En condiciones naturales estas plantas germinan y prosperan mientras los sedimentos están expuestos (Egan y Ungar, 2000), lo que explica la ausencia 14 de ellas en MG. Algunas se mantienen por meses resistiendo la inundación progresiva del humedal y otras desaparecen de la flora establecida e inundada, pero la mayoría, por su carácter anual y malezoide, se mantiene en la zona por la formación de un BS persistente. Los disturbios y la acción humana a la que
Chignahuapan ha sido sometida por décadas, han favorecido la presencia y proliferación de estas plantas y de acuáticas malezoides e invasoras, la abundancia y frecuencia registrada de varias de ellas (Tabla 4) plantea un BS propio de una comunidad vegetal deteriorada y alerta de su eventual proliferación si no se cuenta con programas de manejo y control adecuados.

De acuerdo con Van der Valk et al. (1992) la forma más simple, rápida y económica de restituir la vegetación de un humedal es usando su BS vestigial, y los mejores candidatos para este enfoque son los humedales de agua dulce que tiene fluctuaciones del nivel de agua estacionales o interanuales. En Chignahuapan el BS tiene una riqueza y diversidad considerable, caracterizada por la presencia de especies de alta tolerancia ecológica y la ausencia de acuáticas estrictas anteriormente reportadas como elementos típicos de la región (por ejemplo: Lilaea scilloides, Nymphaea gracilis, Sagittaria latifolia, S. macrophylla y varias especies de Potamogeton; Ramos-Ventura, 1999). Lo anterior junto a la baja similitud de BS con la vegetación establecida sugieren que el BS puede usarse en futuros programas de restauración de la vegetación de la ciénega, con la limitante de restituir principalmente especies de estados sucesionales tempranos. No obstante lo anterior y dada la presencia de diferentes formas de vida y estrategias adaptativas en los elementos que componen el BS, el éxito de su uso dependerá de la selección y ubicación de especies clave, tanto las deseables como de las indeseables (van der Valk et al., 1992), del conocimiento que se tenga de la distribución y los ciclos de vida de estas especies en relación con el ambiente y de la forma en la que el nivel de agua y otros factores ambientales y de perturbación, puedan ser controlados o manipulados para restituir las condiciones ambientales que limitan la auto recuperación.

\section{Agradecimientos}

A la Universidad Autónoma del Estado de México por el financiamiento otorgado y a los revisores anónimos por sus comentarios y aportaciones para mejorar la versión final.

\section{Literatura citada}

Alexander J.M. y D'Antonio C.M. 2003. Seed bank dynamics of French broom in coastal California grasslands: effects of stand age and prescribed burning on control and restoration. Restoration Ecology 11:185-197.

Antón D. y Díaz C. 2002. Sequía en un mundo de agua. Los humedales del alto Lerma. Disponible en: <http://tierra.rediris.es/hidrored/ebooks/sequia/t-cap06.html> (Consultado el 25 marzo, 2014).

Arriaga-Cabrera L., Aguilar-Sierra V. y Alcocer-Durand J. 2000. Aguas continentales y diversidad biológica en México. Comisión Nacional para el Conocimiento y Uso de la Biodiversidad (CONABIO). México D.F. 
Baskin C.C. y Baskin J.M. 2001. Seeds. Ecology, biogeography and evolution of dormancy and germination. Academic Press, San Diego.

Begon M., Harper J.L. y Townsend C.R. 2006. Ecología: individuos, poblaciones y comunidades. Omega, Barcelona.

Bernhardt K.-G., Koch M., Kropf M., Ulbel E. y Webhofer J. 2008. Comparison of two methods characterizing the seed bank of amphibious plant in submerged sediments. Aquatic Botany 88:171-177.

Brown D. 1992. Estimating the composition of a forest seed bank: a comparison of the seed extraction and the seedling emergence methods. Canadian Journal of Botany 70:1603-1612.

Calderón de Rzedowski G. y Rzedowski J. 2001. Flora Fanerogámica del Valle de México. Instituto de Ecología, A.C. y Comisión Nacional para el Conocimiento y Uso de la Biodiversidad (CONABIO), Pátzcuaro.

Clarke P.J. y Dorji K. 2008. Are trade-offs in plant resprouting manifested in community seed banks? Ecology 89:1850-1858.

Colwell R.K. 2009. EstimateS: Statistical estimation of species richness and shared species from samples. Versión 8.2. Disponible en <http://purl.oclc.org/estimates > (Consultado el 20 mayo, 2014).

Cronk J.K. y Fennessy M.S. 2001. Wetland Plants: Biology and Ecology. Lewis Publishers, Boca Raton, Florida.

de Winton M.D. Clayton J.S. y Champion P.D. 2000. Seedling emergence from seed banks of 15 New Zealand lakes with contrasting vegetation histories. Aquatic Botany 66:181-194.

Egan T.P. y Ungar I.A. 2000. Similarity between seed bank and above ground vegetation along a salinity gradient. Journal of Vegetation Science 11:189-194.

Espeland E.K., Perkins L.B. y Leger E.A. 2010. Comparison of seed bank estimation techniques using six weed species in two soil types. Rangeland Ecology and Management 63:243-247.

Espinosa-García F.J. y Sarukhán J. 1997. Manual de Malezas del Valle de México. Universidad Nacional Autónoma de México y Fondo de Cultura Económica, México D.F.

Fenner M. y Thompson K. 2005. The Ecology of Seeds. Cambridge University Press, Cambridge.

Fisher J.L., Loneragan W.A., Dixon K. y Veneklaas E.J. 2009. Soil seed bank compositional change constrains biodiversity in an invaded species-rich woodland. Biological Conservation 142:256-269.

Forcella F. 1992. Prediction of weed seedling densities from buried seed reserves. Weed Research 32:29-38.

Guo Q.F., Rundel P.W. y Goodall D.W. 1998. Horizontal and vertical distribution of desert seed banks: patterns, causes, and implications. Journal of Arid Environments 38:465-478.

Gross K.L. 1990. A comparison of methods for estimating seed numbers in the soil. Journal of Ecology 78:1079-1093.

Haag R.W. 1983. Emergence of seedlings of aquatic macrophytes from lake substrates. Canadian Journal of Botany 61:148-156.

Hammer Ø., Harper D.A.T. y Ryan P.D. 2001. PAST: Paleontological statistics software package for education and data analysis. Paleontología Electrónica 4:9pp. Disponible en: <http:// palaeo-electronica.org/2001_1/past/past.pdf $>$ (Consultado el 5 junio, 2014).

Hopfensperger K.N. 2007. A review of similarity between seed bank and standing vegetation across ecosystems. Oikos 116: $1438-1448$.

Ishikawa-Goto M. y Tsuyuzaki S. 2004. Methods of estimating seed banks with reference to long-term seed burial. Journal of Plant Research 117:245-248.

Kalamees R., y Zobel M. 2002. The role of the seed bank in gap regeneration in a calcareous grassland community. Ecology 83:1017-1025.

Kimber A., van der Valk A.G. y Korschgen C.E. 1995. The distribution of Vallisneria americana seeds and seedling light requirements in the Upper Mississippi River. Canadian Journal of Botany 73:1966-1973.

Leck M.A. 2003. Seed-bank and vegetation development in a created tidal freshwater wetland of the Delaware River, Trenton, New Jersey, USA. Wetlands 23:310-343.

Liu G.-H., Zhou J., Li W. y Cheng Y. 2005. The seed bank in a subtropical freshwater marsh: implications for wetland restoration. Aquatic Botany 81:1-11.

Lot A. y Novelo A. 1978. Laguna de Tecocomulco, Hidalgo. Guía Botánica de Excursiones en México. Sociedad Botánica de México A.C. México D.F.

Lot A., Novelo-Retana A., Olvera-García M. y Ramírez-García P. 1999. Catálogo de Angiospermas Acuáticas de México. Hidrófitas estrictas emergentes, sumergidas y flotantes. Cuadernos Núm. 33. Instituto de Biología, Universidad Nacional Autónoma de México, México D.F.

Lu Z.-J., Li L.-F., Jiang M.-X., Huang H.-D. y Bao D.-Ch. 2010. Can the seed bank contribute to revegetation of the drawdown zone in the Three Gorges Reservoir Region? Plant Ecology 209:153-165.

Magurran A.E. 2004. Measuring Biological Diversity. Blackwell Publishing, Oxford.

McFarland D.G. y Shafer D.J. 2011. Protocol consideration for aquatic plant seed bank assessment. Journal of Aquatic Plant Management 49:9-19

Mesgaran M.B., Mashhadi H.R., Zand E. y Alizadeh H.M. 2007. Comparison of three methodologies for efficient seed extraction in studies of soil weed seed banks. Weed Research 47:472-478.

Moreno C.E. 2001. Manuales y Tesis SEA. Vol 1. Métodos para Medir la Biodiversidad. CYTED, ORCYT-UNESCO y Sociedad Entomológica Aragonesa, Zaragoza..

Pérez O.G. y Valdez M. 2006. El uso de la biodiversidad en las ciénegas del Lerma. En: Cotler-Ávalos, H., Mazari-Hiriat M. y de Anda-Sánchez J. Eds. Atlas de la Cuenca Lerma Chápala: construyendo una visión conjunta. Instituto Nacional de Ecología (INE-SEMARNAT) y Universidad Nacional Autónoma de México, México D.F.

Piudo M.J. y Cavero R.Y. 2005. Banco de semillas: comparación de metodologías de extracción, de densidad y de profundidad de muestreo. Publicaciones de Biología De la Universidad de Navarra, Serie Botánica 16:71-85.

Ramos-Ventura L.J. 1999. Estudio de la flora y la vegetación acuáticas vasculares de la cuenca alta del Río Lerma, en el Estado de México. Tesis de Maestría, Facultad de Ciencias, Universidad Nacional Autónoma de México. México, D.F. 146 pp.

Ruíz M. 2009. Publicación Técnica No. 77. El análisis del Tetrazoleo en el control de calidad de semilla. Ediciones INTA (Instituto Nacional de Tecnología Agropecuaria), Buenos Aires.

Sculthorpe C.D. 1985. The Biology of Aquatic Vascular Plants. Koeltz Scientific Books, Konigstein.

SEMARNAT (Secretaria del Medio Ambiente y Recursos Naturales). 2002. Norma oficial mexicana NOM-059-ECOL-2001. 
Protección ambiental: Especies nativas de México de flora y fauna silvestre. Categorías de riesgo y especificaciones para su inclusión, exclusión o cambio: Lista de especies en riesgo. Diario Oficial de la Federación. 6 de marzo de 2002.

Simpson R.L., Leck M.A. y Parker V.T. 1989. Seed banks: General concepts and methodological issues. En: Leck M.A., Parker V.T. y Simpson R.L. Eds. Ecology of Soil Seed Banks. pp. 283305. Academic Press, Nueva York.

StatSoft, Inc. 2004. Statistica for Windows (data analysis software system), version 7.0. StatSoft, Inc. Tulsa.

Tsuyuzaki S. y Masaki G. 2001. Persistence of seed bank Ander thick volcanic deposit, twenty years after the eruption of Mount Usu, Hokkaudo Island, Japan. American Journal of Botany $\mathbf{8 8}$ : 1813-1817.

van der Valk A.G. y Davis C.B. 1978. The role of seed banks in the vegetation dynamics of prairie glacial marshes. Ecology 59:322-335.

van der Valk A.G., Pederson R.L. y Davis C.B. 1992. Restoration and creation of freshwater wetlands using seed banks. Wetlands Ecology and Management 1:191-197.

Zar J. 1999. Biostatistical Analysis. Prentice Hall, New Jersey.

Zepeda-Gómez C., Lot-Helguera A., Antonio-Nemiga X. y Madrigal-Uribe D. 2012a. Florística y diversidad de las ciénegas del río Lerma, Estado de México, México. Acta Botanica Mexicana 98:23-49.

Zepeda-Gómez C., Antonio-Nemiga X., Lot-Helgueras A., y Madrigal-Uribe D. 2012b. Análisis del cambio del uso del suelo en las ciénegas de Lerma (1973-2008) y su impacto en la vegetación acuática. Investigaciones Geográficas, Boletín del Instituto de Geografía, UNAM 78:48-71.

Recibido: 9 de septiembre de 2014

Aceptado: 15 de noviembre de 2014 